\title{
Ascenso y descenso del proyecto de la paidología rusa a principios del siglo $\mathrm{XX}^{1}$
}

\author{
Elena Minkova \\ Departamento de Psicología y Pedagogía \\ Universidad Pedagógica Estatal de Nóvgorod
}

Rusia

Recibido: 4 de abril de 2014 / Aprobado: 20 de mayo de 2014

Este artículo discute una pregunta que no deja de confrontarnos: ¿por qué la ciencia de la paidología que empezó a desarrollarse en Rusia a principios del siglo XX decayó después de tan solo tres décadas? Y este fenómeno no solo ocurrió en Rusia, sino en otros países que estaban desarrollando esta nueva ciencia. Se presentan los aportes de los lideres de la paidología rusa y también las razones por las cuales fueron objeto de una crítica demoledora. Se concluye que los cientificos rusos fueron personas que se adelantaron a su época y que su aporte debe ser tomado en cuenta en nuestros días en la re-creación de una ciencia similar que se dedique al estudio del periodo de la infancia y las peculiaridades de ese periodo desde diferentes perspectivas.

\section{paidología / ideología / metodología}

\section{Rise and decline of the project of Russian pedology at the beginnings of the 20th Century}

In this article the author discusses the question of the reasons for the rise and decline of the science of pedology that began to develop in Russia at the turn of the $20^{\text {th }}$ Century and decayed only three decades after. This problem did not affect only Russian pedologist, but foreign scientists as well. Included are the notable contributions made by the leaders of the new science of pedology, and also the strong and demolishing criticisms they were subjected to. The conclusions show that the Russian pioneers of pedology were ahead of their time and their contributions ought to be taken into account nowadays in the aim of recreating a similar science that will study infancy and the peculiarities of a child's development from different perspectives.

pedology / ideology / methodology

1 Traducción del inglés por Ricardo Braun.

Correo electrónico: helensea59@mail.ru 


\section{INTRODUCCIÓN}

En el mundo moderno de la literatura psicológica muchos trabajos fueron dedicados al famoso paidólogo ruso Lev Vygotsky, publicados tanto por autores rusos como extranjeros. Sin lugar a dudas, la teoría de Vygotsky es muy conocida en el ámbito del desarrollo infantil, pero sus trabajos pedagógicos continúan siendo subestimados. Habiendo analizado los estudios existentes de la historia de la psicología, podemos asumir que no hay suficiente evidencia de que el tema de la formación de la paidología como una ciencia emergente en Rusia a principios del siglo XX haya sido explorado o inclusive mencionado. Esta conclusión explica la elección del tema para este artículo.

La necesidad de comprender el camino histórico de la paidología en Rusia es importante por las siguientes razones:

a. Extender el campo de la historiografía con la incorporación de datos acerca de la formación de la paidología en Rusia a principios del siglo $\mathrm{XX}$, tomando en cuenta las características socioeconómicas y el trasfondo cultural.

b. Destacar la importancia de la construcción de relaciones de las diferentes escuelas psicológicas del pasado y proporcionar una evaluación adecuada de sus logros.

c. Proporcionar una perspectiva diferente acerca de los problemas con- temporáneos de la integración de la ciencia.

Utilizamos como base de nuestro estudio la investigación cualitativa. Empleando una perspectiva histórica, se permite discutir eventos pasados y presentes en el contexto de la condición actual, y reflexionar y proporcionar posibles respuestas para los problemas y temas de la actualidad.

La metodología sistemática para esta investigación cualitativa es la Teoría Fundamentada (TF). Otro método usado en este estudio es el de la comparación constante, que nos permite contrastar las obras de renombrados psicólogos, con el fin de detectar similitudes y diferencias, así como las razones de su ocurrencia.

Este estudio pretende ilustrar el uso del método de comparación constante como un marco de referencia para la organización y análisis de los datos de la investigación cualitativa. Para ello se proporcionará una breve introducción al uso del método, en la que se examinan procesos como los de categorización, análisis inductivo y el refinamiento de los bits de datos y categorías.

\section{Metodología}

\subsection{Categorización de los sujetos}

La Biblioteca Nacional Rusa en San Petersburgo contiene 833 libros sobre paidología, publicados entre 1904 y 1936. La mayoría de los estudios sobre la psique del niño, que aparecieron en 
ese tiempo, pueden ser clasificados en los siguientes grupos: libros de texto sobre paidología, métodos y programas de paidología, etapas cronológicas apropiadas del desarrollo mental, anormalidades en el desarrollo mental, orientación profesional, y el rol del ambiente en el desarrollo mental de la infancia.

Se puede observar, en la figura 1 , las seis categorías en porcentajes. Dicha figura ilustra que la mayor parte de los libros tratan sobre el rol del ambiente en el desarrollo infantil (40,6\%). Se explica el gran número de trabajos en esta materia por la finalidad principal que tenía la paidología como una nueva ciencia emergente, cuyo objetivo era la creación de una persona con una nueva formación. De acuerdo con la posición de eminentes paidólogos como Vygotsky, Blonsky, Basov y otros, los seres humanos dependen del contexto sociocultural. La explicación del comportamiento humano no radica en las profundidades del cerebro o el alma, sino en las condiciones de la vida externa, y más aún, en las condiciones externas de su vida social y en su forma de existencia sociohistórica.

Otro porcentaje alto corresponde a los libros acerca de los métodos y programas de paidología (23,0\%), lo cual puede explicarse porque la paidología como ciencia estaba en su etapa formativa. Como resultado, las preguntas acerca del objeto de estudio y los métodos de investigación eran centrales para los paidólogos y provocaron muchos debates.

\section{Figura 1}

Porcentajes de libros publicados, según el tema, en el periodo de 1904 a 1936

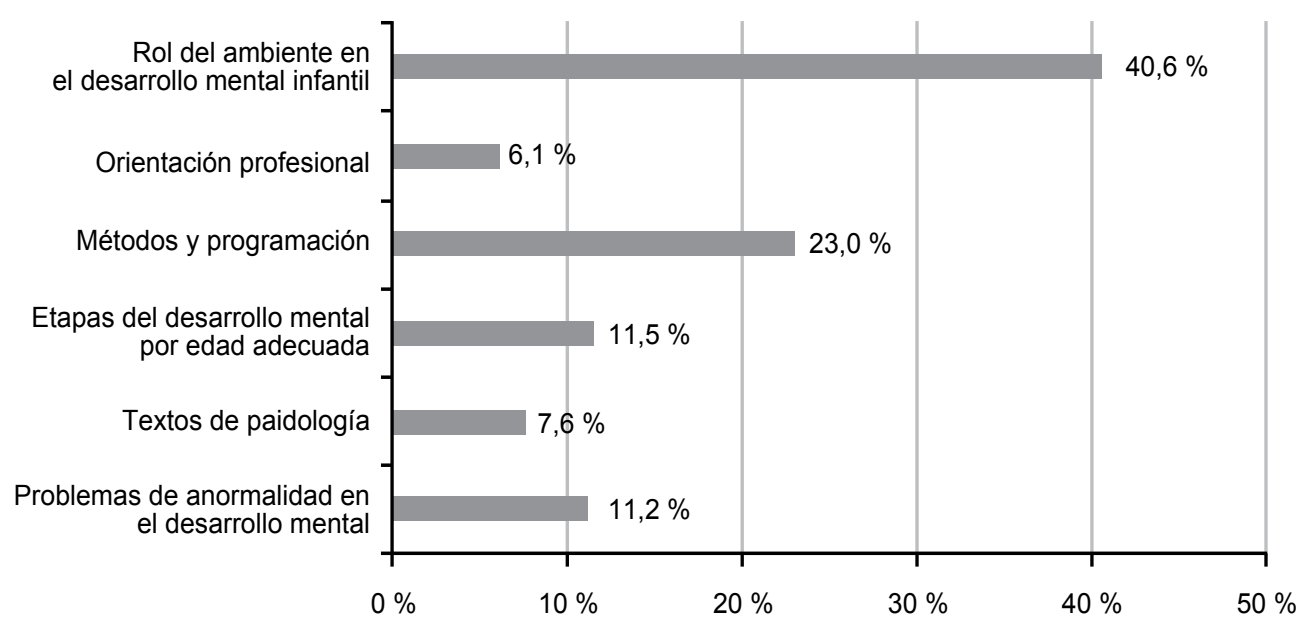

Fuente: Biblioteca Nacional Rusa de San Petersburgo. 
Un porcentaje promedio de los trabajos en paidología estuvieron relacionados con los problemas de las anormalidades en el desarrollo mental (11,2\%). Esto se debe al hecho de que entre los paidólogos había muchos profesionales médicos. La mayoría de libros dedicados a las etapas adecuadas del desarrollo mental (11,5\%) también fueron escritos por médicos. El pequeño número de libros acerca de la orientación profesional $(6,1 \%)$ se explicaría debido a que aquellos problemas solo fueron desarrollados por los paidólogos de ese periodo.

\subsection{La dinámica del desarrollo de la paidología como ciencia en el contexto del tiempo}

La primera etapa, que data de 1904 a 1922, fue el periodo de formación de la paidología como una nueva rama del conocimiento psicológico en Rusia. Por ello existe una lenta producción de libros.

La segunda etapa va de 1923 a 1930, y fue el periodo en el que la paidología alcanzó la cumbre, con un crecimiento dramático del número de obras publicadas sobre esta ciencia.

Figura 2

La dinámica del desarrollo de la paidología como ciencia entre 1904 y 1936

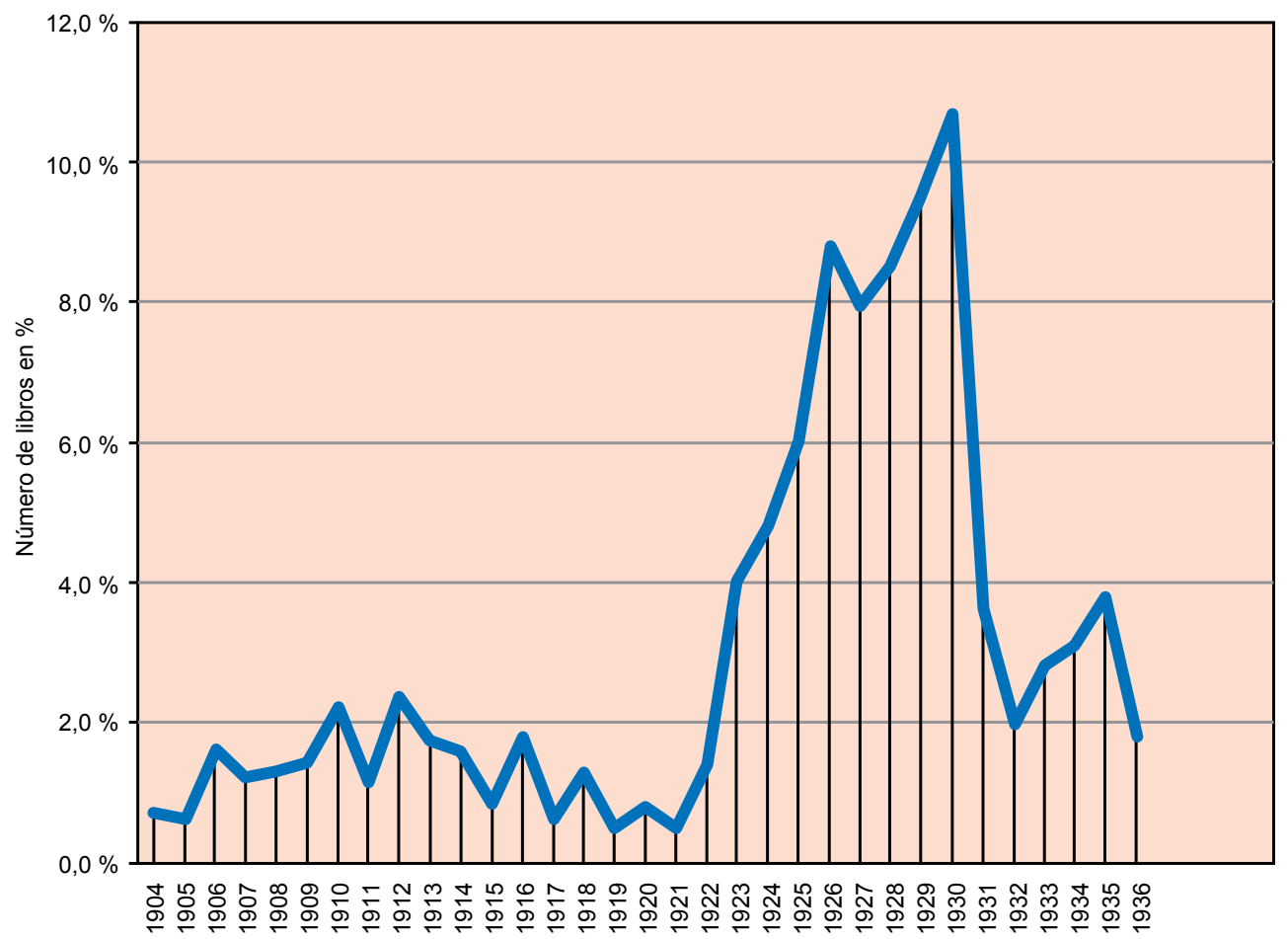

Elaboración propia. 
La tercera etapa, que comprende de 1931 a 1936, se caracterizó por ser un periodo de estancamiento y, en general, se produjo un declive sustancial en el número de publicaciones sobre paidología.

La figura 2 muestra que se puede destacar tres etapas en el desarrollo de la paidología como ciencia, en el periodo entre 1904 y 1936.

\subsubsection{La primera etapa en el desarrollo de la paidología}

En 1904, Nechaev se convirtió en el pionero de los primeros cursos de paidología en Rusia, que se orientaban a estudiar al individuo como sujeto de la educación. Los asistentes a las clases no solamente eran profesores, sino también padres de familia. Estos cursos fueron enseñados por especialistas altamente calificados que tenían un gran potencial académico. Las asignaturas de los primeros cursos paidológicos fueron: Pedagogía, Psicología General, Estudio de Niños con Defectos, Psicofisiología, Psicología Infantil, Anatomía, Patología Nerviosa y Mental, Higiene Infantil, Antropología Criminal, Psicología Comparativa, entre otras.

Una de las clases más populares entre los estudiantes era la del profesor A. A. Krogius (1871-1933). Su tesis doctoral versó sobre El mundo espiritual de los ciegos. Krogius investigó el proceso de memorización de los ciegos utilizando el método de H. Ebbinghaus. De acuerdo con sus observaciones, en todos los casos los ciegos memorizaban textos mejor que las personas con visión. Los estudios de Krogius fueron muy bien conocidos por sus colegas del Oeste. Impartió conferencias en los Congresos Internacionales de Psicología en Innsbruck, Roma y Gotinga.

En 1908, A. Bernstein inauguró en Moscú un laboratorio similar al de $\mathrm{Ne}$ chaev. En febrero de 1917, estaban funcionando los siguientes laboratorios:

- El Laboratorio de Psicología Experimental y Neurología Infantil en el Instituto Neurológico de A. J. Kozhevnikov, bajo la dirección de G. I. Rossolimo.

- La Estación Experimental Médica y Pedagógica de Moscú, bajo la dirección de V. P. Kashenko (posteriormente, se convirtió en la Clínica Médica y Pedagógica de Moscú).

- El Instituto Psicológico en la Segunda Universidad Estatal de Moscú, bajo la dirección de G. I. Chelpanov.

- El Instituto Pedagógico Central, bajo la dirección de N. A. Ribnikov (1921).

En la primera década del siglo XX, empezaron a desarrollarse los primeros programas dedicados al estudio de la infancia. Uno de los programas más completos y populares fue el propuesto por Grigory Rossolimo (1860-1928), denominado el método de los "perfiles psicológicos” (Rossolimo, 1910).

El método de los "perfiles psicológicos" de Rossolimo era un programa 
multidimensional para estudiar la personalidad y consistía en un número de métodos psicológicos experimentales. Resumiendo sus resultados, la persona que investigaba podía obtener una comprensión extensa de las características individuales de un niño o niña en particular. La originalidad del método radicaba en la compilación de las tareas de tests y la manera como se procesaban los resultados. Los resultados del estudio se presentaban en forma de esquema visual, lo que permitía el análisis del individuo examinado. Rossolimo derivó una fórmula que transformaba los datos visuales en datos aritméticos. A mediados de 1920, este método era utilizado en más de 150 laboratorios en toda Rusia. Asimismo, el método del "perfil psicológico" se conoció en el extranjero. De hecho, fue utilizado por Lipmann, Claparède, Schulze, Gieze y otros.

Durante esta primera etapa ocurrieron en Rusia algunos acontecimientos muy traumáticos. En 1905, la Revolución rusa provocó una numerosa pérdida de la población. En 1914, el país entró en la Primera Guerra Mundial y en 1918 empezó la guerra civil rusa. Estos eventos trajeron, además, nuevas víctimas: hubo un incremento en el número de niños sin hogar, y para los adolescentes era muy duro enfrentar la pérdida de sus seres queridos. La paidología condujo un número de estudios acerca del estado emocional de los infantes y adolescentes de ese periodo.
Los trabajos concluyeron que eran los adolescentes los que estaban en mayor riesgo, puesto que tales cambios provocaron un significativo aumento en los intentos de suicidio.

Este problema fue enfrentado por un médico general, E. Radin, quien desarrolló un cuestionario dirigido a estudiantes de diferentes instituciones educativas de San Petersburgo. La investigación sugería que la gente joven estaba muy decepcionada de sus propias vidas, en general. Además, reveló dos tipos de decepción: agresividad y apatía. La causa principal de la apatía era el deseo de provocar fuertes sensaciones y como resultado solo obtenían el sufrimiento del distanciamiento emocional. En otros casos, la caracterización era el aislamiento solitario de cualquier forma de comunicación individual; perdían la habilidad de construir relaciones con otras personas (Radin, 1913).

Los paidólogos también advirtieron del creciente índice de criminalidad en la infancia. Gindes concluyó que eran dos las causas principales del comportamiento criminal. La mayor parte de la criminalidad juvenil era el resultado del ambiente socioeconómico en el que esas personas tuvieron que vivir. El crimen era un "producto social" causado por el ambiente y la crianza. Para Gindes, la causa principal de la delincuencia juvenil era la situación de los jóvenes sin hogar: "La libertad irrestricta de la que gozan los sin techo los destruye, los corrompe y promueve 
el crimen antinatural en una edad de transición" (Gindes, 1923).

La emergencia de la paidología en Rusia estuvo también ligada a la situación socioeconómica y política del país a principios del siglo XX. La Unión Soviética se había embarcado en la construcción de un gran Estado con fuerte poder. Tal Estado necesitaba educar al "nuevo humano", que no solo restauraría la economía, sino también construiría un nuevo gobierno comunista. En consecuencia, la paidología, como una nueva ciencia, tenía la tarea de resolver este problema y cumplir una importante orden gubernamental.

\subsubsection{La segunda etapa en el desarrollo de la paidología}

El año 1923 marcó el punto de partida en el que la paidología floreció, hasta alcanzar la cumbre en 1930. Se produjo un marcado incremento de publicaciones, de $1,4 \%$ en 1922 a 4,0 \% en 1923 , con el mayor número de publicaciones de $10,7 \%$ en 1930.

El rápido crecimiento de la paidología se explica por el siguiente hecho: en 1928 ya habían abierto los siguientes establecimientos académicos en Moscú:

- El Instituto Nacional de Investigación para la Pedagogía Científica en la Segunda Universidad Estatal de Moscú (1926, A. P. Pinkevich).

- La Academia de Educación Comunista (1923, con tres laboratorios pedagógicos: S. S. Molozhavy, P. P. Blonsky, A. B. Zalkind).

- El Instituto Estatal de Psicología Experimental (1924, K. N. Kornilov).

- El Departamento de Salud Infantil del Hospital para Enfermedades Nerviosas de la Primera Universidad Estatal de Moscú (1923, G. I. Rossolimo).

- El Instituto Nacional de Higiene Social de la Intendencia Popular de Salud (1923, A. V. Molkov).

- Los cursos universitarios en la Segunda Universidad Estatal de Moscú (1924, A. B. Zalkind).

- El Instituto de Investigación de Enfermedades Ocupacionales (1923, L. S. Bogolepova).

Asimismo, una serie de importantes eventos paidológicos tuvieron lugar en esta segunda etapa:

- El Primer Congreso Ruso Psiconeurológico (1923).

- El Segundo Congreso de Protección Legal y Social de Menores (1923).

- El Segundo Congreso Ruso Psiconeurológico (1924).

- El Primer Congreso Ruso de Maestros (1925).

- La Segunda Conferencia de Toda la Unión Paidológica (también conocida como la "Reunión Paidológica") (1927).

- El Congreso de la Unión Paidológica (1928).

- El Primer Congreso de Toda la Unión sobre el Estudio de la Conducta Hu- 
mana (también conocido como el “Congreso Conductual") (1930).

- El Tercer Congreso Ruso de Beneficencia Infantil (1930).

Creemos que fue durante este periodo en el que la paidología tenía como finalidad cumplir una tarea de orden social en el Partido Comunista Ruso, esto es, la creación de una nueva psicología marxista y leninista. Debería notarse que al inicio de la segunda etapa los paidólogos genuinamente creían que el ambiente social podía cambiar la naturaleza biológica de una persona. Para muchos paidólogos, el fracaso de la doctrina paidológica se hizo evidente mucho después y produjo una decepción enorme en sus esperanzas y expectativas.

N. K. Kornilov desarrolló una perspectiva radicalmente distinta. Sostenía que cada persona es el producto de su ambiente social. Presentó los lineamientos de su propuesta en su reporte Psicología moderna y marxismo, con ocasión del Congreso Psiconeurológico de 1923, lineamientos que se basaban en los principios que tenían que ser usados para construir la nueva psicología soviética (Kornilov, 1924). Anunciaba que la naturaleza de los procesos mentales solo admitía el único punto de vista verdadero del materialismo. En su discurso en el Congreso, Kornilov manifestó una aguda crítica a la psicología positivista empirista occidental, por ser sumamente subjetiva, individualista y que no reflejaba la verdadera realidad. Según Kornilov, la psicología empírica era el estudio de aspectos aislados, no integrados de la psique humana, tal como, por ejemplo, "habilidad", "memoria", "atención" y otros. Creía que la psicología marxista, en contraste, estaba dirigida a explicar el desarrollo personal y sus propiedades más importantes de manera integral, es decir, tomando en cuenta que dependen de la influencia del ambiente social. Se debe aclarar que no toda la psicología occidental fue objeto de rechazo por parte del entonces líder de la psicología soviética, Kornilov. Aceptaba algunas ideas de la psicología conductista norteamericana, en particular, la doctrina de Watson. Sin embargo, Kornilov argumentaba que era necesario añadir al concepto de Watson los factores sociales, además de los factores biológicos que afectaban el comportamiento humano. Kornilov se convirtió en la cabeza del Instituto de Psicología de Moscú, e inició el cambio de nombre del instituto para convertirlo en Instituto de Reactología. El tema principal del instituto fue la "investigación de la psicología indígena de los proletarios de Moscú a través del método de la determinación del índice de reacción" (Petrovsky, 2007). No hay duda de que el tema principal era la agenda política del Partido Bolchevique. En efecto, no había diferencia alguna en la velocidad de reacción de los proletarios que vivían en Moscú y los proletarios de otras 
ciudades. Sin embargo, ninguno de los miembros del instituto se atrevía a hablar en contra del tema designado. De esta forma, la psicología tenía que servir a los requerimientos ideológicos del nuevo Estado.

Esto se evidenciaba en el creciente número de estudios en los que se analizaba el rol de la situación social en el desarrollo infantil. Estos cambios se asociaron a una nueva ola de ideas comunistas: formar a una nueva persona en una nueva sociedad bajo diferentes condiciones sociales. Se creía que el carácter de una persona joven era determinado por el tipo de trabajo en el que estaba involucrada. El factor industrial era el prevalente, convirtiéndolo en el valor "constante", mientras que el género y la edad era considerados "variables". Los psicólogos creían que para entender el comportamiento de la gente joven, tenían que analizar primero cada aspecto de sus condiciones de vida.

Uno de los fundadores de la paidología fue P. P. Blonsky (1884-1941), quien era profesor en la Universidad de Moscú. Blonsky era una persona muy conocida en el gobierno soviético. Trabajó con la esposa de Lenin, Nadezhda Krúpskaya, en la sección de educación científica del Consejo Académico del Estado. De acuerdo con Blonsky, la paidología estudiaba un complejo de síntomas en las diferentes fases y etapas de la infancia, en una secuencia temporal y con dependencia de las diferentes condiciones ambientales (Blons- ky, 1934). Hacia finales de la década de 1930, Blonsky estaba decepcionado de la paidología, y debatía extensamente con defensores sociogenetistas. Afirmaba que las etapas del desarrollo humano determinadas por la naturaleza no podían ser mejoradas bajo la influencia del ambiente social. Blonsky fue uno de los maestros de Vygotsky (1896-1934), quien estudió en la Universidad de Shanyaysky en Moscú. De acuerdo con el concepto de Vygotsky, el ambiente era la fuente del desarrollo. Formuló una serie de leyes sobre el desarrollo mental de la infancia: la ley de metamorfosis, la ley de las diferencias de indicación de tiempo y ritmo, la ley del desarrollo de las funciones mentales superiores, entre otras.

Los últimos años de la segunda etapa estuvieron marcados por el fuerte control ideológico del Partido Bolchevique sobre las actividades de los científicos. Como respuesta a las demandas del partido, la psicología tenía que prestar especial atención a la influencia del ambiente social en la infancia. Según Joravsky, aun cuando algunas escuelas psicológicas discutían, en el fondo, lo que el partido quería eran teorías que fueran o aspiraran a ser objetivas, materialistas y deterministas (Joravsky, 1989).

La Intendencia de la Educación organizó una reunión de paidólogos en Moscú en 1927 que provocó la crisis de la paidología como ciencia. Los temas principales que se tocaron en esa 
reunión fueron: el rol del ambiente, la herencia y el desarrollo físico; y la importancia de la sociedad como un factor en la modelación de la personalidad infantil. Hubo también una gran controversia acerca de los métodos utilizados en la investigación paidológica. Como resultado de las discusiones, la posición de los sociogenetistas, como Zalkind y Zaluzhny, se impuso sobre las opiniones de los demás. En 1929, tuvo lugar el Primer Congreso Paidológico, donde presentó su trabajo el líder metodológico en paidología, A. B. Zalkind (18881936). Zalkind se había graduado en medicina en la Universidad Estatal de Moscú. Desde 1917 a 1920 fue director del Instituto Psicoterapéutico de Petrogrado. Después de 1925 fue forzado a separarse del psicoanálisis y públicamente se arrepintió de sus "conexiones" con el freudianismo, dirigiendo sus aspiraciones científicas hacia la solución de algunos problemas del desarrollo en la paidología. En 1930 encabezó el Instituto de Psicología, Paidología y Psicotécnica en Moscú.

En su discurso en el Primer Congreso de Paidología, Zalkind instó a los científicos a empezar a construir una escuela de paidología y evitar cualquier lucha o disensión en la ciencia. Por supuesto, este enfoque forzó a los científicos a observar una corrección política rígida, como se observa en sus trabajos. Esto condujo a que la paidología se convirtiera en una sirvienta de la política estatal, lo que significaba que no había libertad de expresión en la ciencia y la búsqueda de la verdad estaba prohibida. A partir de 1929, empezó un acoso hacia todos los científicos cuyas ideas estaban en desacuerdo con el entorno político del Partido Bolchevique.

\subsubsection{La tercera etapa en el desarrollo de la paidología}

La tercera etapa se caracterizó por ser un periodo de estancamiento de la paidología, lo que empezó en 1931. La abrupta caída del número de publicaciones de $10,7 \%$ en 1930 a 3,6 \% en 1931 puede ser explicada por el incremento de la presión ideológica del Partido Bolchevique hacia los paidólogos, que condujo finalmente a una decepción general de la paidología como ciencia.

En nuestra visión, el año 1930 marcó un punto de quiebre para la paidología; los siguientes seis años fueron tiempos de esfuerzos inútiles para la mayoría de científicos que intentaron probar científicamente las ideologías gubernamentales de forma teórica y práctica. En el Congreso de la Unión sobre el Estudio Humano, realizado en Leningrado en 1930, se politizó aún más a la paidología. Para la paidología empezó una "caza de brujas", comenzando por las páginas de la revista Paidología, editada por Zalkind, donde científicos como Arkin, Bechterev, Shelovanov y otros fueron extensamente criticados por sus trabajos (Babushkin, 1932; Feofanov, 1932; Gelmont, 1931; Leventuev, 1931). 
Entre 1929 y 1931, el brillante trabajo de Basov, Principios generales de paidología, fue objeto de duras críticas por su perspectiva abstracto-formal del estudio de la mente de los infantes. M. J. Basov (1892-1931) fue un estudiante de Lazursky. De 1924 a 1931 trabajó como profesor de Paidología y Psicología en el Instituto Estatal de Paidología en Leningrado y en el Instituto Pedagógico de Leningrado, con el nombre de Herzen. Entre 1920 y 1930, se le acusó de ser un antimarxista y, para cambiar su nombre, tuvo que dejar la ciencia y emplearse como aprendiz de mecánica. Después de una pequeña herida en la fábrica, esta le causó infección sanguínea y murió en 1931. En su lecho de dolor, Basov le pidió a su esposa que educara a su hija para que fuera una fuerte y leal comunista. Es importante anotar que Basov fue el primero en subrayar la importancia de "la persona", que juega una parte activa en la construcción del ambiente. Esta idea se desarrolló posteriormente por Vygotsky.

En 1936 se anunció una nueva regulación por el Comité Central Ruso del Partido Comunista de los Bolcheviques, llamada Distorsiones paidológicas en el sistema de los Comités Nacionales de Educación. De acuerdo con estas nuevas reglas, la paidología fue declarada una ciencia burguesa reaccionaria. El Partido Bolchevique creó una serie de tareas para las científicos, una de las cuales fue la de criticar todos los trabajos sobre la teoría de la paidología que habían sido publicados hasta el año 1936.

Un año después de la publicación de esta regulación, comenzó a aparecer un gran número de artículos que criticaban a los paidólogos (Ruskin, 1937; Rubinshtein, 1937; Rudneva, 1937; Svadkovsky, 1937; Zaluzhny, 1937). Svadkovsky llamó a la paidología "la sirvienta del capitalismo" (Svadokovsky, 1937), con ello pretendía hacer notar que esta ciencia tenía la intención de justificar las políticas educacionales de los nazis, que consideraba que la educación solo debía estar disponible para los escogidos. Svadkovsky lo decía porque, debido a la investigación paidológica, cientos de niños fueron clasificados como personas con retardo mental, y solo un grupo pequeño de niños "normales" podían recibir una educación completa.

Los métodos de tests que emplearon los paidólogos provocaron acalorados debates y provocaron la mayor crítica. Zaluzhny sostenía que los métodos de investigación habían sido desarrollados para servir como una justificación de la inequidad de los seres humanos y la raza humana (Zaluzhny, 1937). Es más, señaló las diferencias fundamentales en los propósitos de los estudios paidológicos. La paidología burguesa estaba investigando para poder explotar a los individuos y utilizarlos para su ventaja, mientras que en Rusia los intereses de cada individuo venían primero $y$, por ello, era importante enriquecer a las personas con conocimientos y téc- 
nicas, puesto que sin ellos no podían convertirse en grandes comunistas (Zaluzhny, 1937).

Como resultado de estos acontecimientos, cesaron los trabajos en psicología del desarrollo por muchos años. Fue recién en 1948 que aparecieron los primeros trabajos sobre psicología infantil escritos por Ananjev y Leontjev (Ananjev, 1949; Leontjev, 1948).

Muchos paidólogos tuvieron vidas muy difíciles. Por ejemplo, Nechaev fue acusado y condenado por ser un agitador y propagandista antisoviético, y deportado a Kazajistán en 1935. Pasó los últimos años de su vida en una pequeña ciudad llamada Semipalátinsk y murió en 1948. Zalkind, en cambio, sirvió lealmente al partido y al gobierno, criticando a los paidólogos y sus trabajos. Sin embargo, cuando leyó la nueva regulación de 1936 en el diario, murió de un ataque cardiaco camino a su casa.

\section{La razón para la eliminación de la PAIDOLOGÍA EN RUSIA}

La paidología como ciencia llegó gradualmente a una crisis no solo por la presión ideológica del Partido Bolchevique. Tal como ocurre con cualquier ciencia emergente, la paidología tenía gran número de problemas metodológicos sin resolver. Esto fue expresamente aseverado por Vygotsky en su reporte durante el Encuentro de Psicotécnicos en 1931. Vygotsky creía que la causa de la crisis y el fin de la paidología en el
Oeste y en América fue el empirismo radical que abrazó la paidología inicial. Sostenía que la paidología, como ciencia independiente acerca del desarrollo infantil, solo podía formarse metodológica y prácticamente a través de la comprensión dialéctica y materialista de su objeto de estudio. Vygotsky identificó dos criterios para definir el objeto de estudio de la paidología:

1. La complejidad como una necesidad para comprender los procesos internos en el desarrollo infantil.

2. Desarrollo, entendido por Vygotsky no como expresión única del punto de vista genético, sino como un principio explicativo, es decir, el desarrollo y su esencia interna debían servir como el objeto de investigación de la paidología (Vygotsky, 2010).

Existen diversas hipótesis para explicar la eliminación de la paidología como ciencia. De acuerdo con una de ellas, la paidología terminó con la muerte de los líderes expertos en ella, a saber, Basov, Vygotsky, Lazursky y Rossolimo (Brushlinky, 2000; Petrovsky, 2007). Algunos autores asumen que la paidología fue prohibida porque estaba liderada por Nadezhda Krúpskaya, personaje muy odiado por Stalin (Abul'hanova-Slavskaya et al., 1997; Petrovsky, 2007). De acuerdo con otra hipótesis, algunos paidólogos le tomaron una batería de tests al hijo de Stalin y le otorgaron una escala baja de desarrollo mental (Berezin, 1994). 
Sin embargo, todas estas hipótesis tienen poca evidencia y concuerdo con la opinión de Yaroshevsky, quien afirmó que la eliminación de todo este campo científico fue equivocadamente atribuida a los caprichos y circunstancias azarosas de la vida personal de Stalin (Yaroshevsky, 1994).

Todas las instituciones paidológicas fueron cerradas en 1936 luego que fue puesta en rigor la nueva regulación y se despidió a los paidólogos. A continuación, se destruyeron todas las obras publicadas. La prensa se encargó de hacer una firme crítica en contra de la paidología. Por ejemplo, Ruskin acusó a paidólogos descalificados como responsables de un nivel pobre de diagnóstico del desarrollo intelectual infantil que convirtió a la repetición del año escolar en un fenómeno masivo en la escuela soviética.

En Leningrado, del año 1935 a 1936 , cerca del $14 \%$ de estudiantes de 7 a 13 años tuvo que repetir sus años escolares. Ruskin escribió que la razón prevalente para este fenómeno no era la pobreza hereditaria o las circunstancias de la vida, como lo afirmaba el paidólogo Blonsky, sino los estándares pobres de los mismos docentes (Ruskin, 1937). Sin embargo, las acusaciones contra Blonsky eran infundadas. En el reporte de Blonsky, denominado Los estudiantes que repiten un grado en la escuela, leído en el Congreso de la Unión sobre el Estudio de la Conducta Humana (Blonsky, 1930), espe- cificó una serie de razones para este problema, entre las cuales anotó bajo CI, salud debilitada, capacidad laboral reducida, menor edad comparada con la de otros estudiantes de clase, ambiente social complejo. Adicionalmente, también era un factor la presencia de escolares que provenían de zonas rurales y eran incorporados a las escuelas de la ciudad, quienes resultaban estar muy por detrás de sus pares citadinos (Blonsky, 1930).

Blonsky también recomendó nuevas direcciones para luchar en contra de la repetición de grado:

- Racionalización del sistema escolar (la correcta fusión de clases, la introducción de transferencias semestrales: de una clase a otra, etcétera).

- Organización efectiva del trabajo con los padres de familia y el trabajo extracurricular con los niños.

- Medidas preventivas para mejorar la salud infantil, régimen alimenticio adecuado.

Considero que uno de los factores que más contribuyó a la crisis de la paidología fue el hecho de que el trabajo de los paidólogos en las escuelas adolecía de deficiencias significativas. Muchos paidólogos tenían poca o ninguna formación académica y, por ello, sus investigaciones eran de poca calidad y los resultados obtenidos fueron errados. La idea de la formación de la paidología como una ciencia compleja que estudiara la infancia era ciertamen- 
te ingeniosa. Sin embargo, carecía de una elaboración metodológica adecuada. Aun en nuestros días, se requiere elaborar una ciencia estructurada que pueda cubrir todas las áreas del desarrollo infantil como un todo. Por ello creo que debemos regresar a las raíces de la idea que fundamentó el inicio de la paidología y debemos repensar, reutilizar y re-crear su concepto, que sin duda traerá beneficios al conocimiento actual y la compresión de la niñez en su punto central.

Luego de que la paidología cesara, en general, los hallazgos de las investigaciones no fueron utilizados ni implementados en ningún proceso educacional. Considero que podemos rescatar los principios fundamentales señalados por Petrovsky que cobran actualidad y bien podrían ser usados en la ciencia contemporánea.

De acuerdo con Petrovsky, la paidología se basa en cuatro principios fundamentales:

1. El principio del enfoque holístico en el estudio de la infancia, utilizando datos de las diferentes ciencias.

2. El principio genético, que incluye la idea de zona de desarrollo próximo de Vygotsky.

3. El principio del contexto social, las condiciones de vida del niño o niña.

4. El principio del diagnóstico del nivel de desarrollo infantil, con el propósito de proveer la necesaria asistencia psicológica al individuo y sus padres.

\section{CONCLUSIÓN}

Queda claro que las metas señaladas por los paidólogos a principios del siglo XX estaban adelantadas a su tiempo, y como resultado no pudieron ser realizadas bajo las condiciones socioeconómicas. El proceso de globalización y de las condiciones del mundo contemporáneo hace posible la creación de una ciencia compleja que nos permita estudiar el periodo de la infancia y encontrar las peculiaridades del desarrollo infantil desde diferentes perspectivas.

Muchos de los problemas que la psicología actual trata de resolver ya estaban presentes a principios del siglo XX. El estudio de la historia de la paidología enseña a la psicología contemporánea a tener una actitud cuidadosa con respecto a la infancia, tomando en cuenta las capacidades mentales. En el mundo moderno, por un lado, nadie cuestionaría el hecho de que la psique infantil es versátil $\mathrm{y}$, en consecuencia, necesitamos crear condiciones necesarias para su desarrollo exitoso. Sin embargo, por otro lado, es importante recordar que la mente infantil tiene sus limitaciones, $y$, por lo tanto, requiere un enfoque cuidadoso. Del mismo modo, esto se aplica no solo a la psicología, sino también a otras ciencias, por ejemplo, la ecología, donde se tiende a abusar de los recursos naturales limitados, y al hacer eso, se provoca un daño permanente a nuestro planeta, de la misma forma como si hiciéramos a una niña realizar las grandes expectativas de sus padres. Sostengo 
que las ideas de los psicólogos del pasado pueden ser una fuente de inspiración para nuestros debates actuales. Esto nos enseñará cómo evitar la repetición de los mismos errores y, al mismo tiempo, recordar sus logros. Por ello debemos tener presentes sus nombres y sus ideas. Vivimos en una era en la que debemos resaltar la importancia del conocimiento histórico más que nunca para que nos permita adentrarnos hacia el futuro.

\section{RefERENCIAS}

Abul'hanova-Slavskaya K. A. et al. (1997). Psihologicheskaia nauka v Rossii XX stoletiya: problemi teorii $i$ istorii [Psicología en Rusia en el siglo XX: cuestiones de teoría e historia]. Moscú: Izdatel'stvo "Institut Psihologii RAN".

Ananjev, B. G. (1948). Voprosi detskoi psihologii [Cuestiones de psicología infantil], Moscú, Leningrado: Tip. im. Voroshilova.

Babushkin, A. P. (1932). Eklektika i reakzionnaya kleveta na sovetskogo rebenka ipodrostka [Eclecticismo y calumnia reaccionaria del niño y adolescente soviético]. Pedology, 1-2, 35-41.

Berezin F. B. et al. (1994). Metodika mnogostoronnego issledovaniya lichnosti [El método de estudio multilateral de la personalidad]. Moscú: Folium.
Blonsky, P. P. (1934). Pedologija [Paidología]. Moscú: Gosudarstvennoje uchebno-pedagogicheskoje isdatel'stvo, tip Pech. dvor.

Feofanov, M. P. (1932). Teorija kul'turnogo razvitija $v$ pedologii kak eklekticheskaja koncepcija, imejushaiasa $v$ osnovnom idealisticheskie korni [La teoría del desarrollo cultural de la paidología como un concepto ecléctico que tiene mayormente raices idealistas]. Pedology, 1-2, 21-34.

Gelmont, A. M. (1931). Za marksistsko-leninskuju pedologiju [Para la paidología marxista-leninista]. $\mathrm{Pe}$ dology, 3, 63-66.

Gindes, E. A. (1923). Perehodny vozrast [Adolescencia]. Bakú: Narkompromtorg.

Joravsky, D. (1989). Russian Psychology: A Critical History. Oxford-Cambridge, MA: Basil Blackwell.

Kornilov, K. N. (1924). Sovremennaja psihologija i marksizm [Psicología moderna y marxismo]. Leningrado: Gosudarstvennoje isdatel'stvo.

Leontjev, A. N. (1948). Voprosi detskoi psihologii. [Cuestiones de psicología infantil]. Moscú, Leningrado: Tip. Akademii pedagogicheskih nauk RSFSR.

Leventuev, P. (1931). Politicheskie izvrashenija $\mathrm{v}$ pedologii [Distorsio- 
nes políticas en la paidología]. Pedo$\log y, 3,61-62$.

Petrovsky, A.V. (2007). Psihologija $i$ vremja [Psicología y tiempo]. San Petersburgo: Izdatel'stvo Peter Press.

Radin, E. P. (1913). Dushevnoe nastroenie sovremennoi uchasheisa molodezhi po dannim Peterburgskoi obshestudencheskoi anketi 1912 goda [El estado emocional de la juventud actual de acuerdo con los resultados del cuestionario de estudiantes en San Petersburgo, 1912]. San Petersburgo: Tipographija Karbasnikova.

Rossolimo, G. I. (1910). Psihologicheskij profil'. Kolichestvennoje issledovanije psihicheskih prozessov v norme i patologii. Metodika [Un perfil psicológico. Estudio cuantitativo de los procesos mentales en salud y enfermedad. Métodos]. San Petersburgo: Tipographija Kushnereva.

Rubinshtein, S. L. (1937). Kritike metodov testov [A la crítica de los métodos de examinación]. En Protiv pedologicheskih izvrashenii [En contra de los errores paidológicos]. Leningrado, Moscú: Tip. Pechatny dvor.

Rudneva, E. I. (1937). Pedologicheskie izvrasheniya Vigotskogo [Los erro- res paidológicos de Vygotsky]. Moscú: Gos. uchebno-pedagogich. izd.

Ruskin, L. E. (1937). Pedologicheskije izvrashenija $\mathrm{v}$ voprosah vtorogodnichestva [Errores paidológicos en cuestiones acerca de la repetición de grado]. En Protiv pedologicheskih izvrashenii [En contra de los errores paidológicos]. Leningrado, Moscú: Tip. Pechatnyd vor.

Svadkovsky, I. F. (1937). Krizis burzhuaznoi pedagogiki i lzhenauka pedologia [La crisis de la pedagogía burguesa y la paidología como pseudociencia]. En Protiv pedologicheskih izvrashenii. [En contra de los errores paidológicos]. LeningradoMoscú: Tip. Pechatny dvor.

Vygotsky, L. S. (2010). Pedologia i psihotehnika [Paidología y psicotécnica]. Journal kul'turno-istoricheskoi psihologii, Izdatel'stvo gorodskogo psihologo-pedagogicheskogo universiteta, 2.

Yaroshevsky, M. G. (1994). Marksizm $v$ sovetskoi psihologii [Marxismo en la psicología soviética]. San Petersburgo: Repressirovannaya nauka.

Zaluzhny, A. S. (1937). Lzhenauka pedologia $v$ trudah Zalkinda. [Paidología como una pseudociencia en los trabajos de Zalkind]. Moscú: Izd. Poligrafkniga. 